\title{
Polar properties of a hexagonally bonded GaN sheet under biaxial compression
}

\author{
Yanlin Gao $^{1 *}$, Tomoe Yayama ${ }^{2}$, and Susumu Okada ${ }^{1}$ \\ ${ }^{1}$ Graduate School of Pure and Applied Sciences, University of Tsukuba, Tennodai, Tsukuba, \\ Ibaraki 305-8571, Japan \\ ${ }^{2}$ International Center for Materials Nanoarchitectonics, National Institute for Materials \\ Science, 1-1 Namiki, Tsukuba, Ibaraki 305-0044, Japan \\ E-mail: ylgao@comas.frsc.tsukuba.ac.jp
}

Abstract

Using the density functional theory, we study the geometric and electronic structures of a GaN sheet possessing a honeycomb network. The sheet preserves the planar conformation under an equilibrium lattice constant of $3.2 \AA$, possessing a semiconducting electronic structure with an indirect band gap of $2.28 \mathrm{eV}$. The biaxial compressive strain causes structural buckling, leading to polarization normal to the atomic layer. An external electric field normal to the layer also induces structural buckling whose height is proportional to the field strength. The polarity of the buckled GaN sheet is tunable by attaching $\mathrm{H}$ atoms on $\mathrm{Ga}$ and $\mathrm{N}$ atoms. 
Two-dimensional (2D) materials with atom thickness exhibit peculiar electronic properties arising from their dimensionality and atomic network topology. Because of the bipartite structure of a honeycomb network of carbon atoms, graphene possesses pairs of linear dispersion bands at $\mathrm{K}$ and $\mathrm{K}$ ' points, leading to the vanishing density of states at the Fermi level. In accordance with the linear dispersion bands, graphene possesses extremely high carrier mobility, which allows it to be an emerging material for future electronic, spintronic, optoelectronic, and sensing devices. ${ }^{1-3)}$ Analogous to graphene, other group IV elements, such as silicon and germanium, also form a honeycomb network with structural buckling on appropriate supporting substrates; these structures are known as silicene and germanene, respectively. Despite the structural buckling, free-standing silicene and germanene still maintain similar linear dispersion bands at the Fermi level. ${ }^{4,5)}$ In addition to the honeycomb networks of group IV elements, combination of group III and V elements can form a similar honeycomb network with atom thickness because they have the same valence in the primitive cell of III-V compounds as graphene. Hexagonal boron nitride (h-BN), consisting of boron and nitrogen, which are adjacent to carbon in the periodic table, preserves a planar hexagonal network similar to graphene. Although there are structural similarities of $\mathrm{h}-\mathrm{BN}$ and graphene, $\mathrm{h}-\mathrm{BN}$ is an insulator with a large energy gap of approximately $5 \mathrm{eV}$ because of the electronegativity difference in the constituent elements. $^{6-8)}$

The preceding considerations have stimulated our enthusiasm to explore further variations in 2D materials of III-V compounds. Among the wide variety of possible combinations, gallium nitride $(\mathrm{GaN})$ is a fascinating candidate for $2 \mathrm{D}$ planar materials of III-V compounds, contributing to modern technology as an emerging material. Group III nitride semiconductors with a Wurtzite structure, such as GaN and its derivatives, have attracted much attention because of their excellent optical and electrical properties for applications as optical and high-frequency electronic devices because of their high carrier mobility and wide direct band gap. ${ }^{9-13)}$ For example, InGaN-based quantum well structures have enabled high-performance light-emitting diodes and laser diodes covering from violet to green wavelength regions. ${ }^{14)}$ Furthermore, high-density two-dimensional electron gas at the interfaces in $\mathrm{AlGaN} / \mathrm{GaN}$ heterostructures allows them to be promising materials for high-electron mobility transistors with high voltage and low resistivity. ${ }^{15)}$ In accordance 
with the technological demand, it is natural to consider the possibility of $2 \mathrm{D}$ layered structures of GaN and its derivatives. Şahin et al. have conducted systematic first-principle total energy calculations on the monolayer honeycomb structures of group-IV elementary materials and III-IV compounds and predicted that monolayer $\mathrm{GaN}$ has a planar structure. $^{16,17)}$ Nevertheless, comprehensive and detailed information about the layered structures of $\mathrm{GaN}$ is still absent in the literature and will certainly advance the device technology associated with III-V compounds.

In this study, we aim to investigate the detailed geometric and electronic properties of a sheet consisting of hexagonally arranged $\mathrm{Ga}$ and $\mathrm{N}$ atoms (h-GaN) using first-principles total-energy calculations within the framework of density functional theory and the effective screening medium method. In particular, we theoretically clarify the modulation of the geometric and electronic structure of $\mathrm{h}-\mathrm{GaN}$ using compression, surface hydrogenation, and an external electric field to represent possible applications of h-GaN in electronic and piezoelectric devices. Our calculations showed that the electronic properties of h-GaN are sensitive to biaxial compression and surface hydrogenation: h-GaN is an indirect band gap semiconductor in the planar conformation, while it may be a direct band gap semiconductor in the buckled conformation, depending on the buckling height. Furthermore, because of the chemical difference between $\mathrm{Ga}$ and $\mathrm{N}$ atoms, compressed h-GaN with a buckled conformation possess polarity normal to the sheet, the direction of which is tunable by controlling the surface hydrogenation. In addition, an external electric field normal to the sheet causes the structural modulation of h-GaN from planar to buckled conformations to compensate the electric field by polarization. These facts imply that h-GaN can be applicable for optoelectronic and piezoelectric devices under biaxial strain.

All calculations in this study were performed based on density functional theory implemented in the Simulation Tool for Atom TEchnology (STATE) code. ${ }^{18-20)}$ The generalized gradient approximation with the Perdew-Burke-Ernzerhof functional was used to treat the exchange-correlation potential among interacting electrons, ${ }^{21,22)}$ and the interaction between electrons and ions was described by ultrasoft pseudopotentials according to the Vanderbilt scheme. ${ }^{23)}$ The valence wave functions and charge density were expanded in terms of a plane wave basis set with cutoff energies of 25 Ry and $225 \mathrm{Ry}$, respectively. Structural optimizations were continued until the force on each atom was less 
than $1.33 \times 10^{-3} \mathrm{HR} / \mathrm{au}$. Integration over Brillouin zones was carried out using an equidistance mesh of $4 \times 4 \times 1$ k-points, which give sufficient convergence in the total energy and electronic structure of GaN with hexagonal and Wurtzite structures. We used the effective screening medium method to impose an open boundary condition in the direction normal to the $2 \mathrm{D}$ sheets, allowing us to simulate the polar nature of $\mathrm{GaN}$ under periodic boundary conditions. ${ }^{24)}$ The cell boundary is located above and below the GaN sheet by 4- $\AA$ vacuum spacing. In addition, to investigate the geometric and electronic structure of h-GaN under an electric field, we applied an external electric field normal to the sheet between the two planar electrodes, simulated by the effective screening medium, with infinite permittivity arranged parallel to the cell boundaries. ${ }^{24)}$

Figure 1(a) shows the total energy of monolayer h-GaN as a function of the lattice constant. We can see that the equilibrium lattice constant of monolayer h-GaN is $3.2 \AA$, which is very close to that of bulk GaN with a Wurtzite structure, while the total energy is higher than that of the bulk GaN by $0.87 \mathrm{eV}$ per pair of GaN. Figure 1(b) shows the optimized structure of the monolayer h-GaN under the equilibrium lattice constant; it has a planar hexagonal network consisting of alternating $\mathrm{Ga}$ and $\mathrm{N}$ atoms, similar to that of h-BN. The optimized bond length of GaN is $1.85 \AA$, which is small enough to maintain its planar hexagonal structure because of the substantial interaction between the $p_{\mathrm{z}}$ states of Ga and $\mathrm{N}$ atoms, although the bond length is longer than that of graphene (1.42 $\AA$ ) and h-BN (1.44 $\AA$ ). By carefully checking other possible structures under the equilibrium lattice constant, we confirmed that h-GaN does not possess the buckled structure as in the case of the atomic layers in bulk GaN with a Wurtzite structure, but that the planar structure is the ground state conformation of monolayer h-GaN.

Figure 2(a) presents the electronic structure of $\mathrm{h}-\mathrm{GaN}$ under the equilibrium lattice constant. The h-GaN sheet with the planar conformation is a semiconductor, with an indirect energy band gap of $2.28 \mathrm{eV}$ between the $\mathrm{K}$ and $\Gamma$ points for the valance band top and conduction band bottom, respectively. This makes monolayer h-GaN distinct from bulk GaN with a Wurtzite structure, which has a direct band gap of $2.89 \mathrm{eV}$ at the $\Gamma$ point. ${ }^{25)}$ The calculated effective masses for the electron and hole are $0.96 \mathrm{~m}_{e}$ and $0.51 \mathrm{~m}_{e}$, respectively, which are heavier than those of the bulk GaN, where $\mathrm{m}_{e}$ is the bare electron mass. Furthermore, the electronic band structure of h-GaN is different from that of h-BN, 
which is another planar III-V compound. Figure 2(b) shows the squared wave functions at the $\Gamma$ point for all valence bands and the lowest two branches of the conduction band. The lowest and the doubly degenerated highest valence states at the $\Gamma$ point are primarily distributed on $\mathrm{N}$ atoms with $2 s$ and $2 p_{\mathrm{x}+\mathrm{y}}$ characteristics, respectively. The second lowest state, which corresponds to the valence band top at the $\mathrm{K}$ point, is the $2 p_{\mathrm{z}}$ state of $\mathrm{N}$ atoms. In contrast, by focusing on the conduction band, we find different characteristics for the wave functions: the lowest unoccupied state is mostly contributed by the $4 p_{\mathrm{z}}$ state of Ga atoms, with the $2 s$ state of $\mathrm{N}$ atoms. The second lowest unoccupied state exhibits different characteristics. The state is distributed not only at the atomic sites but also in the vacuum region where atoms are absent (Fig. 2), indicating a nearly free electron state nature that is inherent in layered materials such as graphene and h-BN. ${ }^{26-31)}$ Thus, multilayered h-GaN may exhibit metallic properties similar to graphite intercalation compounds by injecting electrons into this state. ${ }^{32,33)}$

Because strain can effectively tune the physical properties of a material, we studied the strain effects on the geometric and electronic structure of monolayer h-GaN. Figures 3(a) and 3(b) show the buckling height and electrostatic potential difference between $\mathrm{N}$ and Ga surfaces under biaxial compression, respectively. The buckling height is defined as the longitudinal distance between $\mathrm{N}$ and $\mathrm{Ga}$ atoms under compression. The lateral biaxial compression causes structural buckling on the h-GaN sheet, whose height rapidly increases with decreasing lattice parameter. Under a biaxial compression of $6 \%$ (corresponding to $\mathrm{a}=3.0 \AA$ ) or larger, the $\mathrm{h}-\mathrm{GaN}$ sheet prefers the buckled structure, of which the height depends on the compressive strain, rather than the planar conformation. With structural buckling under biaxial compression, polarization normal to the sheet occurs, inducing an internal electric field in the buckled GaN sheet. The polarization induced by the lateral compressive strain implies that h-GaN with the planar conformation undergoes a structural phase transition into the buckled conformation under an external electric field normal to the sheet. Figure 3(c) shows the buckling height as a function of the external electric field. The buckling height is found to monotonically increase with increasing field intensity, reaching $0.18 \AA$ when the electric field is $-1.54 \mathrm{~V} / \AA$. It should be noted that such a large field is hardly applied under usual experimental conditions. In the buckled structure under the electric field, Ga atoms are shifted to negative electrode while the $\mathrm{N}$ atoms are shifted 
to positive electrode. Thus, the $\mathrm{h}-\mathrm{GaN}$ sheet exhibits piezoelectricity and inverse piezoelectricity, being applicable to piezoelectric devices with atom thickness.

The biaxial compressive strain modulates the electronic structure of the h-GaN sheet. The calculated electronic properties of the compressed h-GaN sheet are summarized in Table I. The electronic structure of compressed $h-G a N$ is sensitive to the lateral lattice constant. With decreasing lattice constant, first, the band gap gradually increases, retaining its indirect band gap nature. With a lattice constant of $\mathrm{a}=2.9 \AA$, the sheet is a semiconductor, with a direct gap of $2.92 \mathrm{eV}$ at the $\Gamma$ point. Then, by further decreasing the lattice constant, suddenly, the sheet undergoes a phase transition into a metallic phase in which the valance and the conduction bands touch each other at the $\Gamma$ point. This fact indicates that h-GaN under biaxial strain may be applicable as a constituent material of ultrathin optoelectronic, optical, and photovoltaic devices because of its tunable direct band gap.

Although the h-GaN sheet with the buckled conformation exhibits interesting electronic properties, unsaturated $2 p$ and $4 p$ states of $\mathrm{N}$ and Ga atoms, respectively, cause structural instability without external constraints. To stabilize the h-GaN with the buckled conformation, here, we consider h-GaN with the buckled conformation in which an $\mathrm{H}$ atom is attached to each $\mathrm{Ga} / \mathrm{N}$ atom to saturate their $p$ states. The optimal lattice constant of fully hydrogenated h-GaN with buckled conformation is $3.2 \AA$, which is the same as that of the h-GaN sheet with the planar conformation [Fig. 4(a)]. Under the optimized structure, the sheet shows structural buckling with a height of $0.69 \AA$ [Fig. 4(b)]. Fig. 4(c) shows the calculated energy band structure of the fully hydrogenated h-GaN. The sheet is a semiconductor with a direct band gap of $3.68 \mathrm{eV}$ at the $\Gamma$ point, which is wider than the sheet with the planar conformation and bulk GaN with a Wurtzite structure.

In addition to the substantial stabilization of $\mathrm{h}-\mathrm{GaN}$ in the buckled conformation, hydrogenation of buckled h-GaN sheets causes an inversion of polarity. Figure 5 shows the plane-averaged electrostatic potential of $\mathrm{h}-\mathrm{GaN}$ with clean and hydrogenated surfaces normal to the layer. For the buckled sheet with clean surfaces, the electrostatic potential just outside the $\mathrm{N}$ surface is higher than that outside the $\mathrm{Ga}$, in accordance with the electric field induced by the compressive strain. In contrast, the potential difference between the $\mathrm{N}$ and Ga surfaces of the hydrogenated h-GaN sheet is opposite to that of the buckled sheet 
with clean surfaces. Thus, surface hydrogenation can tune the polarity of h-GaN with the buckled conformation. The inversion of polarity with hydrogenation is induced by positively and negatively charged $\mathrm{H}$ atoms attached to $\mathrm{N}$ and $\mathrm{Ga}$ atoms, respectively: to saturate the valence states of surface $\mathrm{N}$ and $\mathrm{Ga}$ atoms, pseudoatoms with valence electrons of $0.75 \mathrm{e}$ and $1.25 \mathrm{e}$ are necessary, respectively. ${ }^{34)}$ Therefore, the neutral $\mathrm{H}$ atoms on the surface induce excess and deficit electrons on the $\mathrm{N}$ and $\mathrm{Ga}$ surfaces, respectively, resulting in polarity opposite to that of the sheet with clean surfaces. It is also expected that chlorination and fluorination of h-GaN lead to different electrostatic potentials and polarities. $^{35)}$

We studied the geometric and electronic structures of hexagonally bonded GaN sheets under biaxial compressive strain based on first-principles total-energy calculation within the framework of density functional theory and the effective screening medium method. Our calculations showed that the electronic properties of $\mathrm{h}-\mathrm{GaN}$ are sensitive to biaxial compression. The biaxial compressive strain causes structural buckling on h-GaN, which leads to the modulation of the electronic energy band around the gap: h-GaN with the planar conformation is an indirect gap semiconductor, while that with the buckled conformation may be a direct gap semiconductor. The structural buckling causes polarization normal to the sheet because of the electronegativity difference between $\mathrm{Ga}$ and $\mathrm{N}$ atoms. Furthermore, an external electric field normal to the sheet causes the structural modulation of h-GaN from planar to buckled conformations to compensate the electric field by polarization. By attaching $\mathrm{H}$ atoms onto all $\mathrm{Ga}$ and $\mathrm{N}$ atoms, h-GaN preserves the buckled structure, in which the polarity of the sheet is opposite to that of the buckled h-GaN with clean surfaces. These facts imply that h-GaN can be applicable for optoelectronic and piezoelectric devices under biaxial strain.

\section{Acknowledgments}

This work was partly supported by CREST, from the Japan Science and Technology Agency, a Grant-in-Aid for Scientific Research (25246010, 16H00898, 16H06331) from the Ministry of Education, Culture, Sports, Science and Technology of Japan, and the Joint Research Program on Zero-Emission Energy Research, Institute of Advanced Energy, Kyoto University. A part of the calculations was performed on an NEC SX-Ace at the Cybermedia Center at Osaka University and on an a SGI ICE XA/UV at the Institute of 
Solid State Physics, The University of Tokyo.

\section{References}

1) K. S. Novoselov, A. K. Geim, S. V. Morozov, D. Jiang, Y. Zhang, S. V. Dubonos, I. V. Grigorieva, and A. A. Firsov, Science 306, 666 (2004).

2) K. S. Novoselov, A. K. Geim, S. V. Morozov, D. Jiang, M. I. Katsnelson, I. V. Grigorieva, S. V. Dubonos, and A. A. Firsov, Nature 438, 197 (2005).

3) Y. Zhang, Y.-W. Tan, H. L. Stormer, and P. Kim, Nature 438, 201. (2005)

4) S. Cahangirov, M. Topsakal, E. Aktürk, H. Şahin, and S. Ciraci, Phys. Rev. Lett. 102, 236804 (2009).

5) G. G. Guzmán-Verri and L. C. Lew Yan Voon, Phys. Rev. B 76, 075131 (2007).

6) K. Watanabe, T. Taniguchi and H. Kanda, Nat. Mater. 3, 404 (2014).

7) L,Wirtz, A, Marini and A. Rubio, Phys. Rev. Lett. 96, 126104 (2006).

8) R. M. Ribeiro and N. M. R. Peres, Phys. Rev. B 83, 235312 (2011).

9) R. J. Molnar, R. Singh, and T. D. Moustakas, Appl. Phys. Lett. 66, 268 (1995).

10) J. M. Van Hove, R. Hickman, J. J. Klassen, and P. P. Chow, Appl. Phys. Lett. 70, 2282 (1997).

11) Y. Zhao, R. M. Farrell, Y.-R. Wu, and J. S. Speck, Jpn. J. Appl. Phys. 53, 100206 (2014).

12) D. S. Lee, Z. Liu, and T. Palacios, Jpn. J. Appl. Phys. 53, 100212 (2014).

13) T. Kachi, Jpn. J. Appl. Phys. 53, 100210 (2014).

14) H. Zhao, G. Liu, J. Zhang, J. D. Poplawsky, V. Dierolf, and N. Tansu, Opt. Express 19, A991 (2011).

15) X. Liu, Y. Lu, W. Yu, J. Wu, J. He, D. Tang, Z. Liu, P. Somasuntharam, D. Zhu, W. Liu, P. Cao, S. Han, S. Chen, and L. S. Tan, Sci. Rep. 5, 14092 (2015).

16) H. Şahin, S. Cahangirov, M. Topsakal, E. Bekaroglu, E. Akturk, R. T. Senger, and S. Ciraci, Phys. Rev. B 80, 155453 (2009).

17) A. Onen, D. Kecik, E. Durgun, and S. Ciraci, Phys. Rev. B 93, 085431 (2015). 
18) P. Hohenberg and W. Kohn, Phys. Rev. B 136, 864. (1964)

19) W. Kohn and L.J. Sham, Phys. Rev. A 140, 1133 (1965).

20) Y. Morikawa, K. Iwata, and K. Terakura, Appl. Surf. Sci. 169-170, 11 (2001).

21) J. P. Perdew and A. Zunger, Phys. Rev. B 23, 5048 (1981).

22) D. M. Ceperley and B. J. Alder, Phys. Rev. Lett. 45, 566 (1980).

23) D. Vanderbilt, Phys. Rev. B 41, 7892 (1990).

24) M. Otani and O. Sugino, Phys. Rev. B 73, 115407 (2006).

25) K. Miwa and A. Fukumoto, Phys. Rev. B 48, 7897 (1993).

26) M. Posternak, A. Baldereschi, A. J. Freeman, E. Wimmer, and M. Weinert, Phys. Rev. Lett. 50, 761 (1983).

27) M. Posternak, A. Baldereschi, A. J. Freeman, and E. Wimmer, Phys. Rev. Lett. 52, 863 (1984).

28) A. Catellani, M. Posternak, A. Baldereschi, and A. J. Freeman, Phys. Rev. B 36, 6105 (1987).

29) X. Blase, A. Rubio, S.G. Louie, and M.L. Cohen, Phys. Rev. B 51, 6868 (1995).

30) N.-T. Cuong, M. Otani, and S. Okada, J. Phys. Condens. Matter 26, 135001 (2014).

31) A. Yamanaka and S. Okada, Appl. Phys. Express 7, 125103 (2014).

32) T. Enoki, M. Suzuki, and M. Endo, Graphite Intercalation Compounds and Applications (Oxford University Press, Oxford, 2003).

33) M. S. Dresselhaus and G. Dresselhaus, Adv. Phys. 30, 139 (1981).

34) K. Shiraishi, J. Phys. Soc. Jpn. 59, 3455 (1990).

35) M. Xiao, T. Yao, Z. Ao, P. Wei, D. Wang and H. Songa, Phys. Chem. Chem. Phys. 17, 8692 (2015). 


\section{Figure Captions}

Fig. 1. (a) Total energies of monolayer $h-G a N$ as a function of the lattice constant; energies are measured from that of bulk GaN with a Wurtzite structure.

(b) The geometric structure of monolayer $\mathrm{h}-\mathrm{GaN}$ under the optimum lattice constant.

Fig. 2. Band structure and squared wave functions at the $\Gamma$ point of monolayer h-GaN. Energies are measured from that of the valence band top. Band indexes correspond to those of squared wave functions.

Fig. 3. (a) Buckling height and (b) electrostatic potential difference in monolayer h-GaN as a function of the lattice constant. (c) Buckling height of monolayer h-GaN under an external electric field.

Fig. 4. (a) Total energies of hydrogenated h-GaN sheet as a function of the lattice constant. (b) Geometric and (c) electronic structures of the fully optimized h-GaN with a hydrogenated surface.

Fig. 5. Plane-averaged electrostatic potential of h-GaN sheet with (a) clean and (b) hydrogenated surfaces.

Table I. Band gap of compressed h-GaN sheet with different lattice constant.

\begin{tabular}{|c|c|c|c|c|c|}
\hline a $(\AA ̊)$ & 3.2 & 3.1 & 3.0 & 2.9 & 2.8 \\
\hline $\mathrm{E}_{\mathrm{g}}(\mathrm{eV})$ & 2.28 & 2.73 & 3.05 & 2.92 & 0 \\
\hline Gap & indirect & indirect & indirect & direct & metal \\
\hline
\end{tabular}


Template for APEX (Jan. 2014)

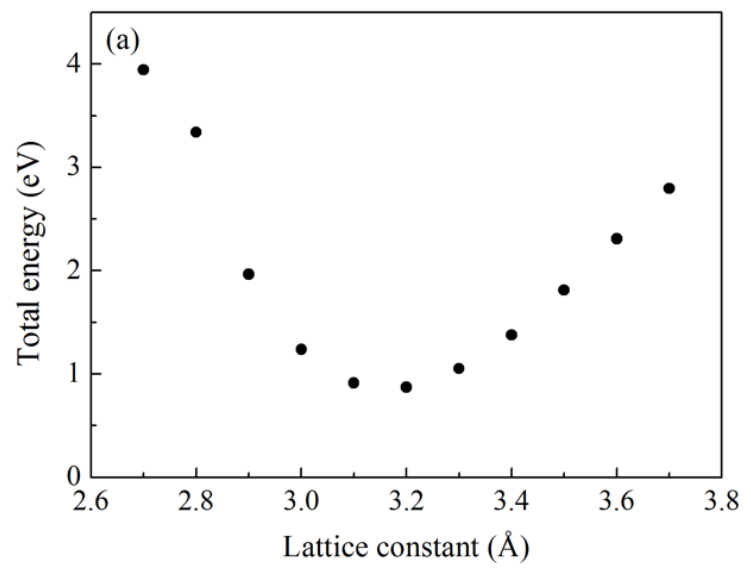

(b)

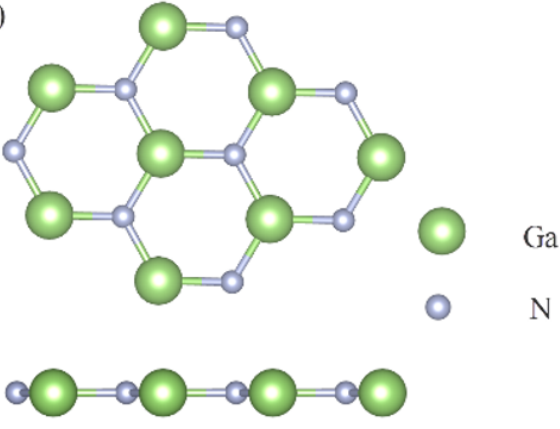

Fig.1. 
Template for APEX (Jan. 2014)

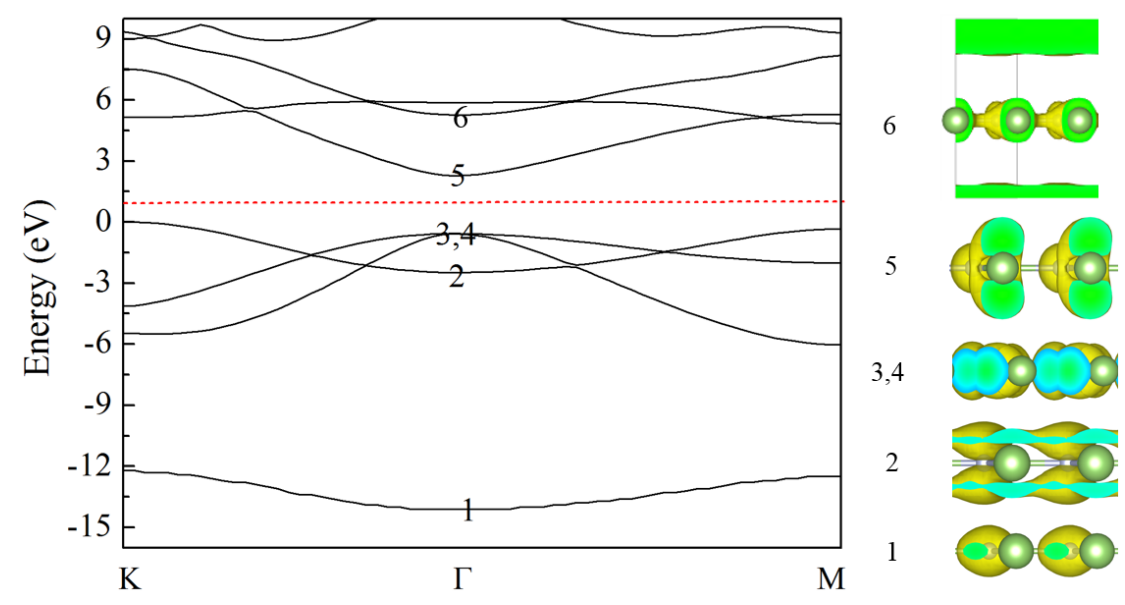

Fig.2. 
Template for APEX (Jan. 2014)
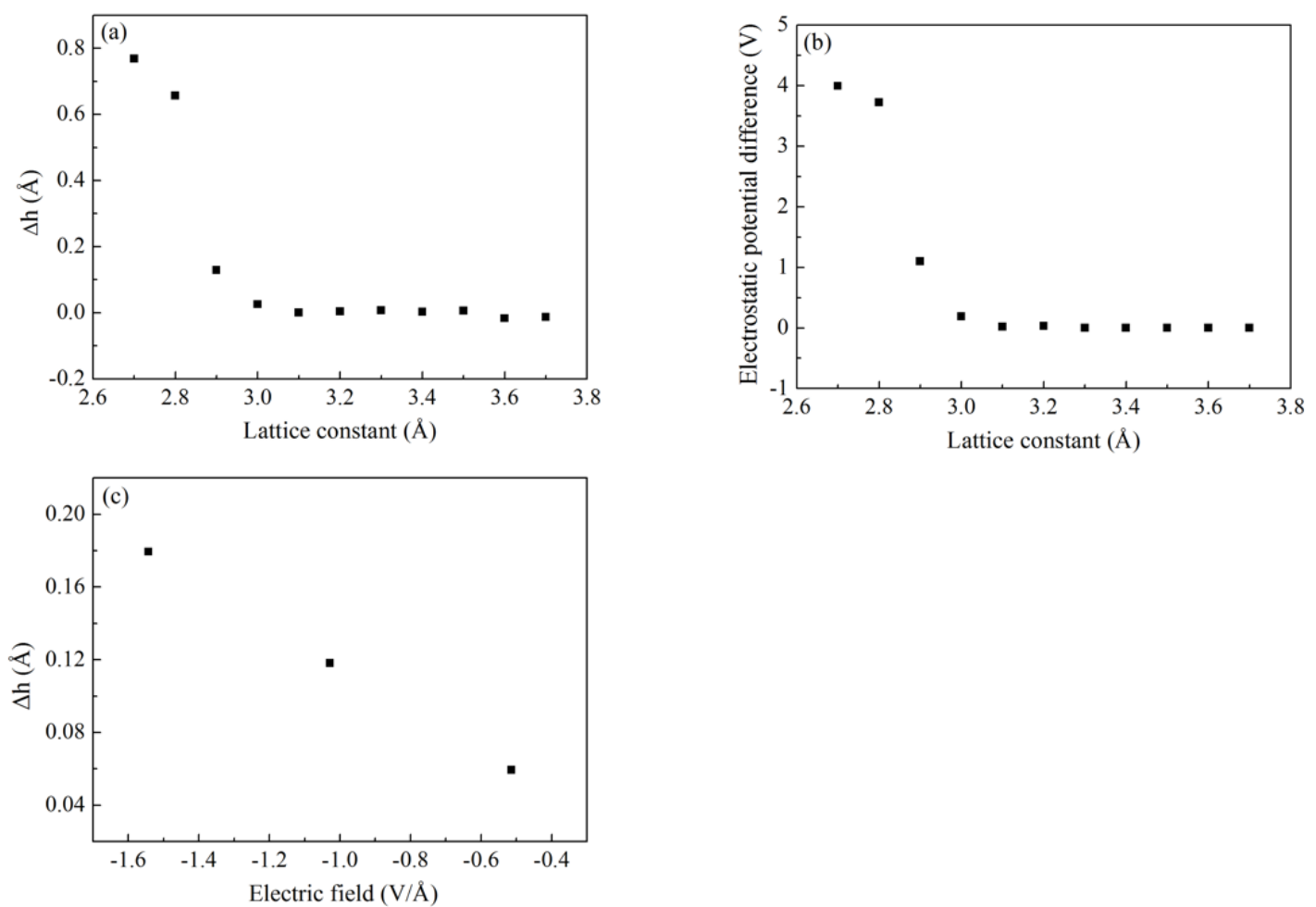

Fig.3. 
Template for APEX (Jan. 2014)
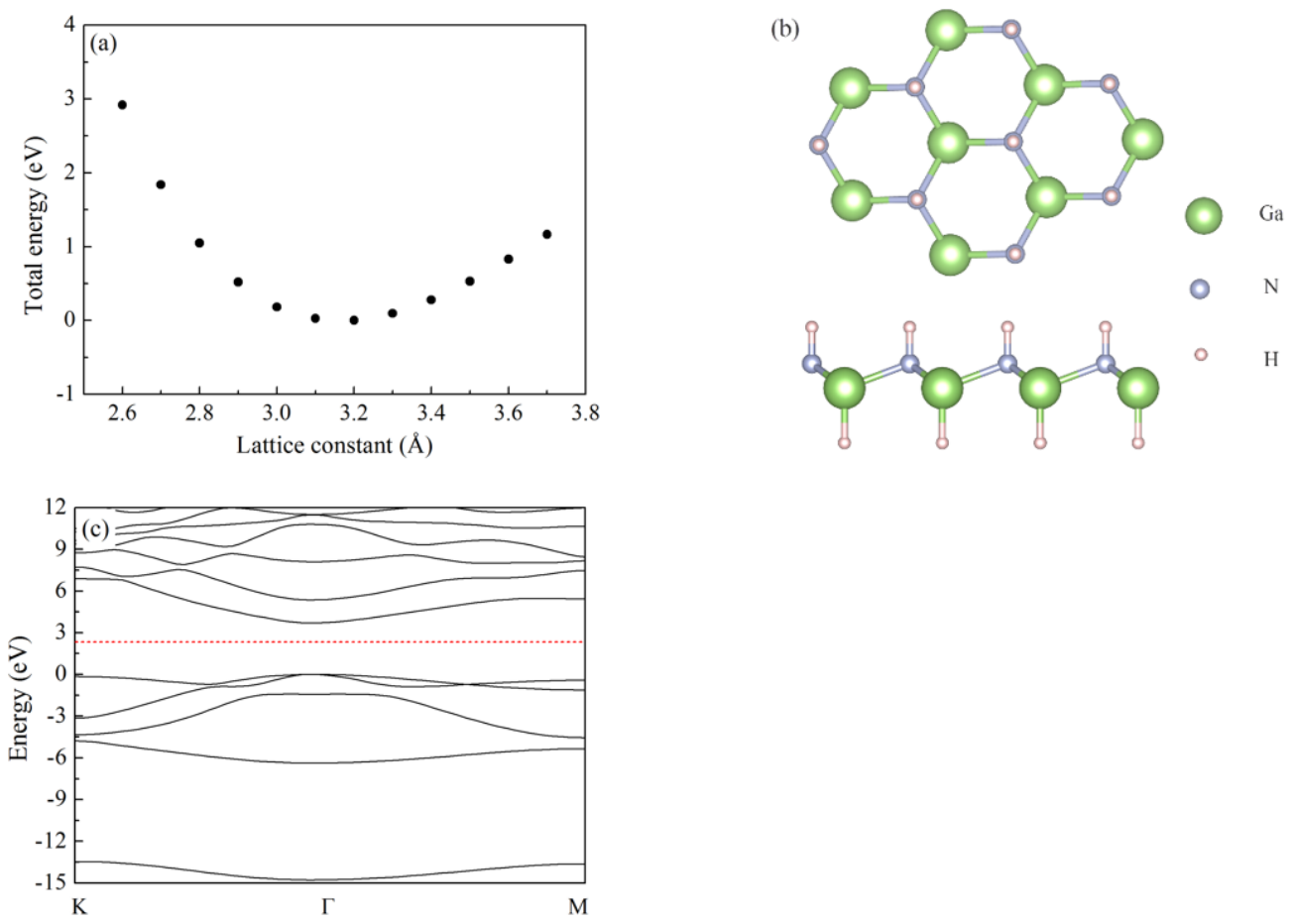

Fig.4. 
Template for APEX (Jan. 2014)
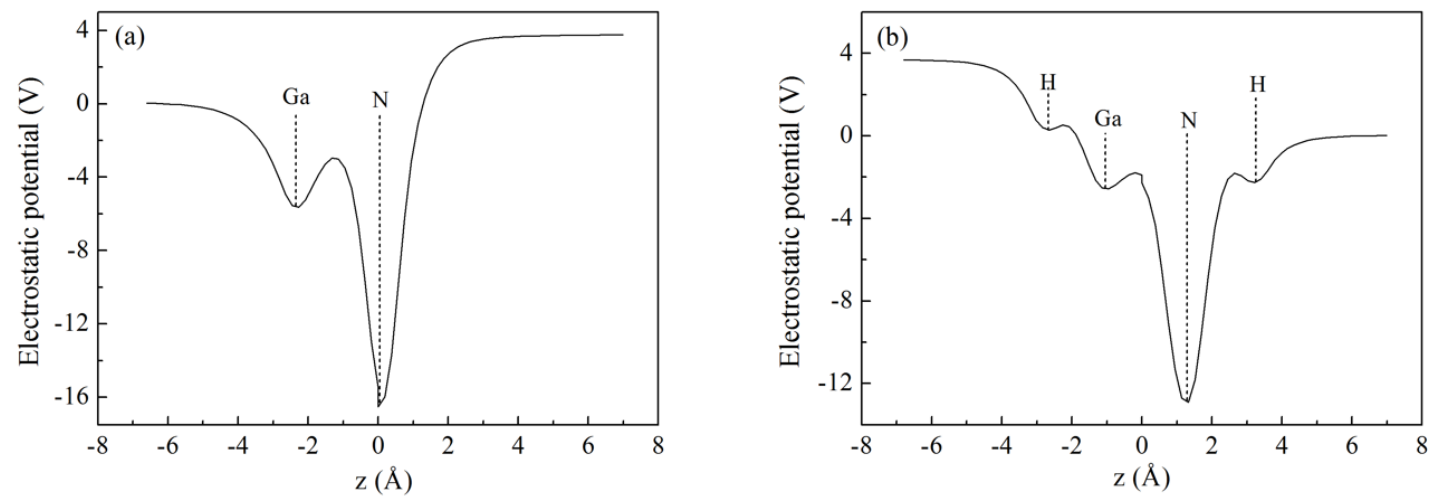

Fig.5. 